\title{
INTERFERENCE CANCELLATION FOR UNDERWATER ENVIRONMENT
}

\author{
B Priyalakshmi, G Dileep Reddy, V Pavan Raju, N Ravi Teja, GSandeep Reddy
}

\begin{abstract}
In this paper, Performance of MIMO-OFDM systems in underwater wireless optical communication are studied. The execution of MIMO underwater environment regulation is examined. To alleviate turbulence induced fading, spatial diversity over underwater environment link is used. Furthermore, scattering and absorption are considered in the analysis. Analytical BER computations are fundamentally the same as simulated results. Interference cancellation inclusion in OFDM system has better BER performance than conventional method. So, by IC can accomplish high BER productivity than conventional system. Moreover, simulated results demonstrate that OFDM using IC plot has better BER execution for underwater environment.
\end{abstract}

Index Terms: Multiple Input Multiple Output, Interference cancellation, Bit Error Rate, Monte Carlo, Orthogonal Frequency Division Multiplexing .

\section{INTRODUCTION}

Lately, the investigation of marine assets has drawn the consideration of all nations on the planet. So as to fulfill the continuous development of human exercises with high-transfer speed information transmission in underwater condition, specialists have proved the idea of underwater networks (UWN). The fundamental UWNs comprise of ocean bed sensors, floats, vehicles which are remotely operated and communication hubs [1]. Highlighting efficiency and low latency the underwater environment frameworks can give rapid transmission to low-range applications, and been viewed as a important integral answer for the long-run acoustic communication in UWSNs. Ocean water indicates generally low constriction to light with a wavelength going from 450 to $550 \mathrm{~nm}$, which compares to the green-blue range. An $8 \mathrm{~m} / 9.6 \mathrm{~Gb} / \mathrm{s}$ underwater environment framework based on $405 \mathrm{~nm}$ blue-light Laser Diode transmission with a 16-QAM OFDM signal was proposed in [2]. While LD based frameworks $h$ been exhibited for broadened extents and large information rate, LED based plans favored by applications requiring minimal effort and consumption of power [3-5].

There are still a few difficulties ahead for underwater environment frameworks, since the optical bar is altogether constricted, inferable from the scattering, absorption impacts of water. Furthermore, marine turbulence will cause

Revised Manuscript Received on July 05, 2019.

B Priyalakshmi, Department of Telecommunication Engineering, SRMIST, 603202, India.

G Dileep Reddy, Department of ECE, SRMIST, 603202, India

V Pavan Raju, Department of ECE, SRMIST, 603202, India.

N Ravi Teja, Department of ECE, SRMIST, 603202, India.

GSandeep Reddy, Department of ECE, SRMIST, 603202, India. scintillation and result in connection outage [6]. One of the promising answers for determination the turbulence issue is MIMO technique, which has been concentrated broadly in optical fiber communication and VLC, due to its capacity to expand the transmission information rate. In addition, MIMO frameworks can likewise give spatial diversity increase to relieve fading, and along these lines to increase the framework execution. The bit error rate execution of MIMO underwater environment was numerically studied, and the practical results demonstrated that MIMO innovation can alleviate the channel turbulence, yet in addition expand the range of communication, particularly for low turbulence channels. The MIMO-OFDM procedure utilizes space array elements to transmit OFDM flags, to improve the resilience to multipath and noise.

In this paper, MIMO-OFDM underwater environment framework, which utilized DC biased optical OFDM, was analyzed. The performance of MIMO OFDM using IC is simulated with the consideration of coastal parameters.

\section{CHANNEL AND SYSTEM MODEL}

In this section, absorption and scattering are taken to model underwater environment channel and hence present the system model in this paper.

\section{A.Underwater Channel}

Absorption or scattering caused by interactions between photon and particles in sea water while optical light propagation. Photons lose their energy when interacted with particles in water. In scattering process, direction alters occurs which is also one of the reasons for energy loss, so receiver aperture captures few photons. Energy loss due to scattering and absorption can be characterized by scattering coefficient $\mathrm{b}(\lambda)$ and absorption coefficient $\mathrm{a}(\lambda)$. Therefore, attenuation coefficient $\mathrm{c}(\lambda)=\mathrm{a}(\lambda)+\mathrm{b}(\lambda)$ [1-8]. In [8], the impulse response of the channel based on MC underwater environment channel. The general instructions of this approach are as follows: photon position; transmission direction by azimuth angle $\varphi$ and zenith angle $\theta$; intensity (i); every photon position introduced at $(0,0,0)$ with unit weight and zero begin time.

Before connecting with the molecules in the water, the photon distance $\mathrm{h}$ calculated by $h=-\ln \delta /(a+b)$, where $\delta$ is random variable between zero and one, and the intensity (i) of the photon will be refreshed as $i^{\prime}=i(1-a / c)$. The direction after scattering $\left(\mathrm{a}_{\mathrm{x}}, \mathrm{a}_{\mathrm{y}}, \mathrm{a}_{\mathrm{z}}\right)$ the direction of the next movement $\left(\mathrm{a}_{\mathrm{x}}{ }^{\prime}, \mathrm{a}_{\mathrm{y}}{ }^{\prime}, \mathrm{a}_{\mathrm{z}}{ }^{\prime}\right) \quad$ is characterized by $\varphi$ and $\theta$ then 
new direction is characterized by [1] [8].

$\mathrm{a}_{\mathrm{x}}{ }^{\prime}=\cos \varphi \sin \theta$

$\mathrm{a}_{\mathrm{y}}{ }^{\prime}=\sin \varphi \sin \theta$

$\mathrm{a}_{\mathrm{z}}^{\prime}=\frac{a_{z}}{\left|a_{z}\right|} \cos \varphi$

So, the new position of photon refreshed as

$\mathrm{x}^{\prime}=\mathrm{x}+\mathrm{a}_{\mathrm{x}}{ }^{\prime} \mathrm{h}$

$y^{\prime}=y+a_{y}^{\prime} h$

$z^{\prime}=z+a_{z}{ }^{\prime} h$

The following ought to be ended, when the photon achieves the collector plane opposite to the $\mathrm{z}$ axis, or the photon's weight is lower than a limit estimation of $10-6$, and just the ones inside the aperture of receiver and with $\theta$ less than the half angle of field of view can be chosen as the received photons. Over and over track until the last photon following is finished. The movement time, position, intensity and direction for every photon are recorded. The gathered load of the identified photons with a similar landing time is standardized by the all-out transmit intensity, which is utilized to show the plot of power versus travel time for unit intensity transmitted, and it is proportional to the impulse response of the channel.

\section{B. System Model}

Consider a MIMO OFDM communication system with nT transmitters and $\mathrm{nR}$ receivers. The input digital data is modulated to get QAM modulated data. The serial QAM modulated output is converted into parallel data. After serial to parallel conversion, the parallel data stream is converted from frequency domain to time domain by IFFT. Then again data stream is parallel to serial converted and fed into D/A convertor to drive the LED transmitters. The optical signals will be sent into an underwater channel.

Consider MC model for modeling the channel characteristics of underwater channel. After going through underwater channel, the signal received by photodiodes. Then output from photodiodes is converted from analog to digital. Received output is serial to parallel converted. Interference cancellation is done before demodulation of the signal.

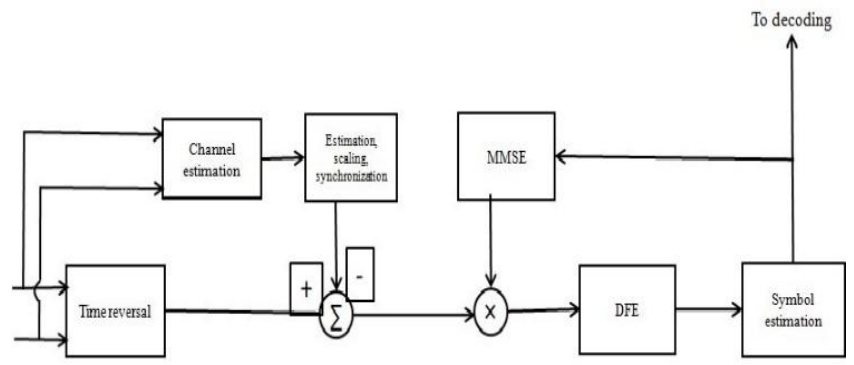

Fig1: Block diagram of interference cancellation technique

While preserving the SNR gain achieved by time reversal preprocessing, we can remove the residual interference experienced by each uses with the introduction of interference cancellation technique. Interference at the receiver can be removed easily by interference cancellation. Decision feedback equalizer can be used for removing inter symbol interference which is similar to interference cancellation. Before reducing the target signal after time reversal combing the estimation can be scaled and synchronized. Before symbol decoding the interference, cancellation is applied as the process is allowed to iterate until a stationary point is reached to improve the estimated symbols. Interference cancellation is characterized by the equation given below

$$
z_{k}^{-}(t)=z_{k}(t)-\sum_{i l \neq k}^{M} \beta_{k 1} \widehat{1_{k l}}\left(t-\widehat{\Delta_{k l}}\right)
$$

\section{SIMULATION RESULTS}

In this section we present the simulation results for bit error rate performance of under- water wireless optical communication in coastal water scenario. In simulating impulse response by Monte Carlo method by considering coastal water parameters [9] as ab- sorption coefficient $=$ 0.088 , scattering coefficient $=0.216$ and attenuation coefficient $=0.305$.

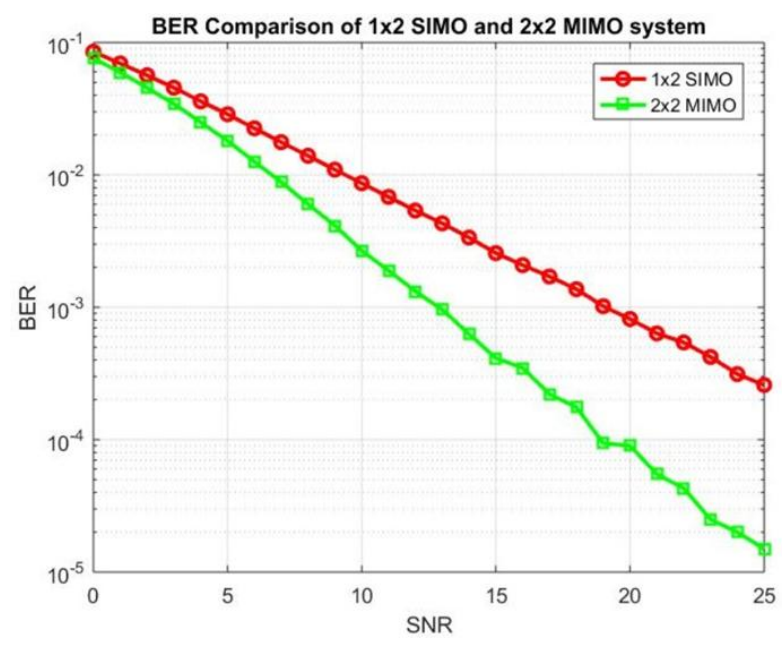

Fig 3.1: BER comparison of MIMO with SIMO scheme

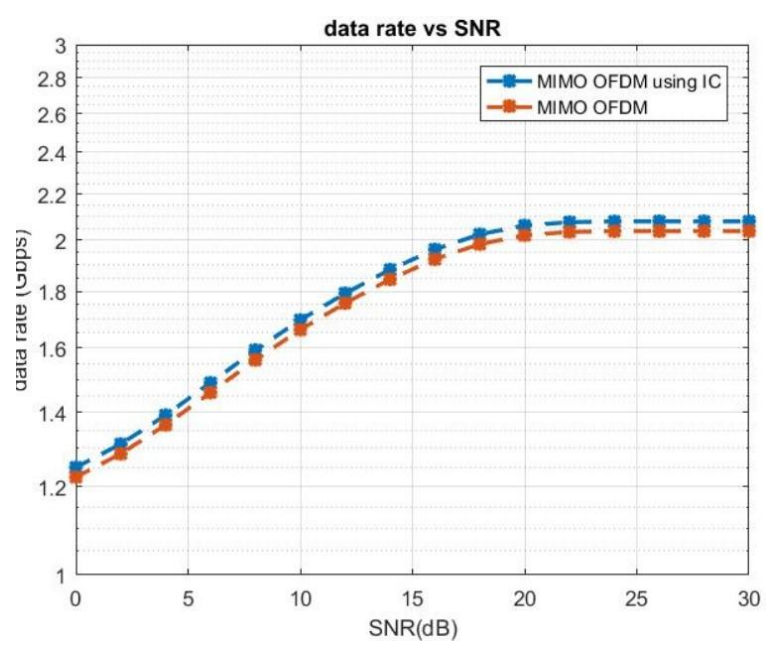

Fig 3.2: Data rate of MIMO OFDM using IC

Fig 3.1. Shows the analysis of bit error rate with signal to noise ratio (SNR) which depicts that BER performance 
of 2x2 MIMO scheme has better performance than 1x2 SIMO scheme. In this analysis only absorption, scattering and turbulence effects are considered. In spite of above underwater parameters there is one parameter i.e., chlorophyll concentration which effects the transmission rate. If this parameter is considered deep analysis of BER vs SNR can be studied. Fig 3.2 shows the data rate achieved by IC scheme and conventional scheme. Data rate of $2 \mathrm{Gbps}$ been achieved. Fig 3.3 depicts BER performance of MIMO-OFDM using interference cancellation ans is better than conventional method. BER range of IC scheme in powerof- 8 been achieved which is better compared to conventional scheme which is in power of-6.

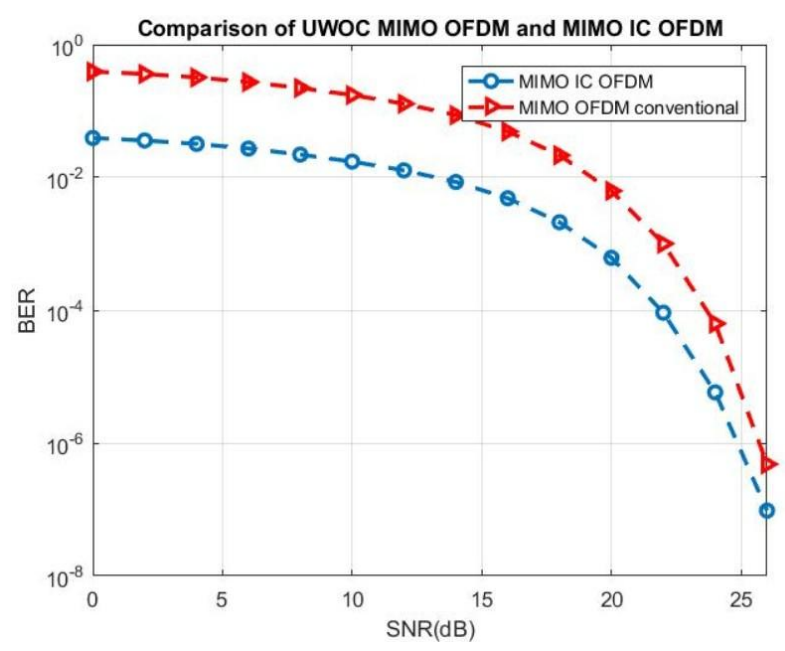

Fig 3.3: BER performance of MIMO OFDM using IC for Underwater

\section{IV.CONCLUSION}

The design presented was shown to improve the bit error rate of conventional OFDM method by using IC technique. As the simulation results shows that MIMO OFDM using IC is better bit error rate performer than conventional MIMO OFDM. To design the system with IC involves complex calculations and bit difficult to implement. Interference cancellation inclusion in OFDM system has better BER performance than conventional method. So, IC can accomplish high BER productivity than conventional system Moreover simulated results demonstrate that OFDM using IC plot has better BER execution for underwater environment

\section{REFERENCES}

1. Johnson, L., Green, R., \& Leeson, M. (2013). A survey of channel models for underwater optical wireless communication.

2. H.-H. Lu, et al., An $8 \mathrm{~m} / 9.6 \mathrm{~Gb} / \mathrm{s}$ underwater wireless optical communication system, IEEE Photonic S J. 8 (5) (2016) 1-7.

3. Doniec, M., Detweiler, C., Vasilescu, I., Chitre, M., Hoffmann-Kuhnt, M. \&Rus, D. (2010). AquaOptical: A Lightweight Device for High-rate Long-range Underwater Point-to-Point Communication. Mar TechnolSoc J, 44(4), 55-65.

4. Cossu, G., Corsini, R., Khalid, A. M., Balestrino, S., Coppelli, A., Caiti, A., \&Ciaramella, E. (2013). Experimental demonstration of high speed underwater visible light communications.
5. Wang, P., Li, C., Wang, B., \& Xu, Z. (2016). Real-Time 25Mb/s Data Transmission for Underwater Optical Wireless Communication Using a Commercial Blue LED and APD Detection.

6. Baykal, Y. (2016). Scintillation index in strong oceanic turbulence. Optics Communications, 375, 15-18.

7. Priyalakshmi B,Mahalakshmi K,Analysis of Suitable Modulation Techniques for Underwater Wireless Optical Communication, Journal of Advanced Research in Dynamical and Control Systems Vol. 10. Sp$13 / 2018$

8. Jasman, F., \& Green, R. J. (2013). Monte Carlo simulation for underwater optical wireless communications.

9. Zeng, et al., A survey of underwater wireless optical communication, IEEECommun. Surv. Tut. 19 (1) (2016) 204-238.

\section{AUTHORS PROFILE}

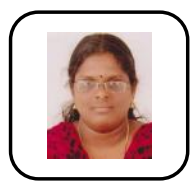

B. Priyalakshmi completed her Bachelor's of Engineering from GCE, Bargur, Madras University (2001), and completed Master's of Engineering from GCT, Coimbatore, Anna University(2004) and MBA from BSA Cresent College, Anna University (2010). At present she is working as an Assistant Professor at SRM Institute of science and Technology, India.

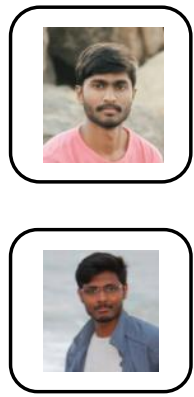

G Dileep Reddy, completed his Bachelor of Technology in the Department of ECE (2018), SRM Institute of science and Technology, 603202, India.

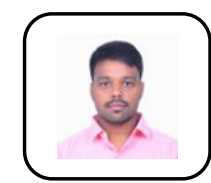

N Ravi Teja, completed his Bachelor of Technology in the Department of ECE(2019), SRM Institute of science and Technology, 603202, India.

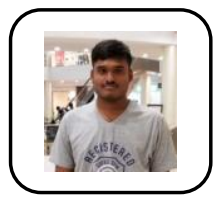

G Sandeep Reddy, completed his Bachelor of Technology in the Department of ECE(2019), SRM Institute of science and Technology, 603202, India 